\title{
Ser-en-el-cuerpo: tentativas para un esclarecimiento sobre cómo aparece un cuerpo. En y mundo
}

\author{
Being-in-the-body: attempts to a clarification about how a body \\ appears. In and world
}

\author{
JUAN JOSÉ GARRIDO PERIÑÁN*
}

\begin{abstract}
Resumen: El cuerpo ha sido un problema para la mayor parte del pensamiento filosófico. En concreto, para el filósofo alemán Martin Heidegger, quien apenas realizó algún comentario sobre el mismo. En nuestra meditación, por tanto, hemos intentado cuestionar, con la ayuda de algunos conceptos heideggerianos, la problemática de la corporalidad humana, entendiéndola como ser-enel-cuerpo. Para ello, hemos comprendido a éste como "un comportarse", en el horizonte fenomenológico de un "en" y un "mundo". En relación a ello, se ha explicitado los equívocos de ciertas concepciones contemporáneas al considerar el cuerpo como significante hegemónico, a fin de proponer, a modo de esbozo, una cierta propedéutica cuya principal tarea sea la de transparentar nuestro propio modo ser. Si transparentamos nuestro modo de ser, esclareceremos la problemática de la corporalidad.
\end{abstract}

Palabras clave: Heidegger; Fenomenología; Cuerpo; Hermenéutica; Ontología.

\begin{abstract}
The body has been a problem for most of philosophical thought. In particular, for the German philosopher Martin Heidegger, who barely made any comment on it. In our meditation, we have tried to question, with the help of Heidegger's concepts, about problem of human corporeality, understanding it as beingin-the-body. To do this, we have understood this being-in-the-body as "a to bear oneself", in a phenomenological horizon which is expressed as "in" and "world". In this way, it has explained the mistakes of some contemporary interpretations which consider the body as hegemonic signifier. Lastly, we try to propose, as an outline, a propaedeutic whose main task is the transparency of our own way of being. If we reveal our way of being, we clarify the issue of corporeality.
\end{abstract}

Keywords: Heidegger; Phenomenology; Body; Hermeneutics; Ontology.

Fecha de recepción: 24/05/2016. Fecha de aceptación: 20/07/2016.

* Esta comunicación ha sido posible gracias al estipendio otorgado por el Ministerio de Educación, Cultura y Deporte (MECD), del Reino de España, en relación a la convocatoria FPU13/00375. Universidad de Sevilla, Investigador Contratado en Formación, jjgarper@us.es. Lineas de Investigación: Fenomenología; Heidegger; Ontología; Hermenéutica. Últimas publicaciones: "El horror a ti debido: lo inhóspito en el origen de la obra de arte de Martin Heidegger", en: Problemata: Revista Internacional de Filosofí, vol. 6., n 2., pp. 283312. [DOI:http://dx.doi.org/10.7443/problemata.v6i2.23310.]; "Un diálogo entre naturaleza y libertad en el horizonte del ser. Kant y Heidegger", en: Endoxa: Series Filosóficas, [DOI: http://dx.doi.org/10.5944/ endoxa.36.2015.13956 ]. 


\section{Introducción}

En la época de la ausencia -o muerte, según Nietzsche- de Dios, una cierta, y dominante, visión fisiológica del cuerpo ha imperado por doquier, hasta el punto de afirmar, en un alarde de fobia metafísica, que somos el conglomerado -quizás azaroso- de millones de células, que, químicamente, pululan transidamente entre nuestros órganos. Que nos componemos de materia es un hecho palmario, pero que somos materia es una afirmación algo más difusa, yo diría, más bien, que ésta, es una afirmación filosófica. Precisamente, somos incapaces de pensar propiamente el sentido de ese somos, pues, aun compartiendo la materia más material con los animales o plantas, nuestro ser parece muy distinto al ser de ellos. Nuestra principal tarea es, por tanto, la dilucidación de ese somos. Para ello propongo pensar ese somos radicalmente, esto es, fenomenológicamente, a fin de esclarecer cómo llega éste a aparecer.

Por otro lado, el problema de tener un cuerpo lo vamos a definir, acudiendo a la fenomenología heideggeriana, en tanto "comportarse" [sich-Verhalten] con respecto a algo que ya (se) es. Afirmar que "somos corporales" es re-aceptar el factum de que estamos siempre ya llevados más allá de nosotros mismos (Cfr. Heidegger, 1989, 110, 118 y 119), es decir, afectados por "temples anímicos" [Stimmungen], temples que para "determinar(nos)los" [Bestimmen], lo tenemos que hacer siempre en un "dejar(nos)-templar" [Stimmen]. El cuerpo es, por ende, una manifestación de nuestro ser-en-el-mundo, un ser-siendo-con-otros, un siendo en una situación fáctica concreta. Por eso, nuestro cuerpo es "cuerpo vivido" [Leib] (Cfr. Husserl, 1952, 145-146; Heidegger, 2006, 116-117 y 1986, 41) y no cuerpo -solamente- material [Körper]. La pregunta directriz sería: ¿es la corporalidad [Leiblichkeit], construida en relación al constructo hegemónico, cultural, político o social, el fundamento de todo comportarnos en el mundo (ser-en-el-mundo), o, contrariamente, la corporalidad es derivada de la posibilidad ontológica de que algo como un mundo se nos abra y a partir de él nos pensemos y seamos?

Responder a esta pregunta, a nuestro juicio fundamental, constituirá el objeto de nuestra aportación.

\section{Primero el mundo, luego el cuerpo}

Qué sea el cuerpo no es la cuestión. La cuestión estribaría en arrinconar las múltiples determinaciones del eidos cuerpo, para responder al horizonte del interrogante: ¿cómo es posible un ser-en-el-cuerpo? Cuando se mienta la palabra cuerpo, se está explicitando el cuerpo del ser humano, el cuerpo material y vivido del mismo, no el cuerpo de un animal, el cuerpo de un maniquí, sino el cuerpo del yo, del tú y del nosotros. Y es que vivimos nuestro cuerpo de forma diferente del animal, de la vaca y del maniquí. Nuestro modo de ser, dijimos, es ser-en-el-mundo. Esto significa que nos comportamos en base a disposiciones afectivas que nos exponen intencionalmente en las cosas que nos rodean, las cosas que "están-ahí" [Vorhandensein]. Precisamente, nos caracterizamos, a diferencia del modo de ser de las cosas particulares, en que, para ser, tenemos que ser junto a aquello que nos condiciona, arrojados a nuestra posibilidad más nuda. Heidegger lo dice más melancólicamente: el modo de ser del aquel que existe, el Dasein, es el ser en "cada caso mío" [Jemeinigkeit], es decir, que en mi posibilidad de ser radica la de dejar de ser. De mi ser, yo no puedo predicar 
un principatum, características impasibles y estáticas que lo enmarquen y definan; antes al contrario, de mi ser sólo puedo decir que radica en "tener que ser" [zu sein], inevitablemente, sin elección. Pero este tremendismo heideggeriano, no sería más que una abstracción si no siguiéramos intuyendo los recovecos de lo explícito en el dictum: ¿tener que ser dónde ${ }^{1}$ ? La respuesta se antoja fácil, pero es esquiva: decimos "en el mundo". De ahí que se afirme que el ser que somos nosotros mismos es un ser "en" el mundo. Todavía no ha quedado claro que quiera decir la preposición "en” (Cfr. Infra., 2.1.), ni qué papel jugará para este ser que nosotros mismos somos, pero, al menos, podríamos aventurar cómo el mundo se esboza como el horizonte "abierto" [offen] que soporta al ente que existe, o vive: el ser humano. El mundo es "el suelo fundante" [der gründende Boden], el único espacio para que la dación de la fenomenalidad se dé, y su horizonte de sentido se abra para el ente que la re-clama: el Dasein. No es de extrañar, por tanto, que Martin Heidegger, en Ser y Tiempo, muestre lo siguiente:

Dos entes, que están en el mundo en el estado de estar-ahí y, aparte de eso, carecen de mundo por sí mismos, no pueden, nunca, tocarse, ninguno de ellos puede estar junto al otro $(1986,55)^{2}$.

En el "carecer de mundo" [Weltles], que escenifica el modo como los entes existen en la "efectividad" [Wirklichkeit], como "puestos-ahí1" [Gegenstand] en la realidad [Realität], se esconde un principio para intentar atisbar la relevancia de la corporalidad en el pensar heideggeriano, pues, según parece, ser-en-el-mundo significa ser "tocado" y "estar junto al otro". Tener un mundo es compartir la posibilidad de la comunión con el amigo, la comunicabilidad con el extranjero, el entendimiento filosófico, el amor de tu pareja, la comunidad política; estar en la brecha del "estado de apertura" [Erschlossenheit] del ser, donde acontece todo venir-a-la-presencia. Este mundo, entendido como horizonte de "aprenhensibilidad" para todo ente, es ontológicamente previo 4 para Heidegger, soportador de toda determinación

1 Habrá que hacer notar, para hacer justicia a la tentativa del propio Heidegger en su proyecto más ambicioso: Ser y Tiempo, que el horizonte que delimita dónde es el Dasein, está insuficientemente pensando en el opus magnum, habiéndose decantado el autor por ahondar en las relevancias del quien es el que tiene que ser. La moraleja es bien fácil: el quien del Dasein es su propio horizonte de temporalidad, pues es en el tiempo donde el ser acaece, se da para el Dasein. La explicitación de este quien es ejercida en la Segunda Sección del Tratado (1986, 251-453).

2 "Zwei Seiende, die innerhalb der Welt vorhanden und überdies an ihnen selbst weltlos sind, können sich nie "berühren”, keines kann bei dem andern sein”. El autor de texto se hace cargo de la traducción. No obstante, conviene no olvidar la excelente traducción del profesor chileno Jorge Eduardo Rivera (1997).

3 Gegenstand es unas de las traducciones al español de objeto. Para Heidegger, Gegenstand viene a traducir la expresión latina objectum y se diferencia de Objekt en que Objekt es pensado como cualquier objeto temático, mientras Gegenstand es el objeto temático determinado previamente como quididad, puesto, predispuesto y a disposición del subiectum.

4 Debería entenderse que el tratamiento de Heidegger a lo previo, está emparentado con aquello que él 1lama lo "pre-teorético" [Vor-theoretisches], esto es, todas aquellas estructuras que describen el modo de ser del Dasein, sus existenciarios: ser-en-el-mundo, el cuidado, la caída, etc. Esto ya es esbozado en 1919, en el conocido curso de posguerra $(1999,59)$ : "Se ha de romper con esta primacía de lo teorético, pero no con el propósito de producir un primado de lo práctico o de introducir otro elemento que muestre los problemas desde una nueva perspectiva, sino porque lo teorético mismo y en cuanto tal remite a algo pre-teorético". ["Diese Vorherrschaft des Theoretischen muß gebrochen werden, zwar nicht in der Weise, daß man einen Primat des Praktischen 
sobre el ente. La corporalidad se asienta, no obstante, a partir de lo abierto en el mundo. Corporalidad es, entre otras cosas, comportarse en el mundo desde un cuerpo material, que siente, padece, piensa, un cuerpo que no es elegido, sino que nos atenemos a él, como el existente a su existencia finita y fáctica. Pero, entonces, ¿la apertura del ser del ente, mediada por el horizonte-mundo, no responde a un mecanismo cultural, de poder, de mediación histórica? ¿No está, nolens volens, Heidegger practicando el uso de su criticada metafísica, es decir, falsando el principio de la diferencia ontológica a favor de una entidad, una substancia (ousia), un principio a priori, impensado e impuesto? Por último, ¿no responde este mundo a una serie de estructuras fundadas históricamente?

\section{El cuerpo creado. La imposibilidad de un esclarecimiento del comportarse}

Hay una corriente de pensamiento bastante extensa, practicada desde la segunda mitad del siglo XX, que considera que todo lo que es estriba en una convención humana, un producto, cultural e histórico, que responde a estructuras de voluntad de poder ${ }^{5}$. Al socaire de la crítica a la metafísica -considerada ésta como esencialista- ejercida por Heidegger, la mayoría de estos pensadores se han esforzado por describir genealógicamente la estructura de formación de estos conceptos que, en su mayoría, actúan como significantes de identidad. Bien es verdad, como en el caso del feminismo, que esta corriente de pensamiento ha servido para transparentar, acaso radicalmente, la socavación en la que, a lo largo de la tradición, se ha visto la mujer ${ }^{6}$, por ejemplo, enclaustrada en una identidad de sumisión y vasallaje. Para ello se han destruido las construcciones hegemónicas de conceptos tales como el de sexo y género, se ha abierto la clausura de todo eidos, al considerarlo impotente, vago, infértil 7 . Con la idea de cuerpo ha sucedido algo similar, pues al cambiar los mecanismos de anclaje de identidad en los se había asentado nuestra imago, se ha producido una modificación de nuestro ser-en-el-cuerpo. ¿Es esta modificación de nuestra corporalidad (ser-en-el-cuerpo) actual, un fenómeno originario? ¿o, más bien, esta modificación del comportarnos junto a nuestro cuerpo, sigue la estructura ontológica del modus vivendi moderno y, por tanto, es una consecuencia cultural o social?

Que hayamos descubierto, en nuestro tiempo posmoderno, que los conceptos son productos de una convención humana, y que detrás de ellos nada se esconda, no quiere,

proklamiert, und nicht deshalb, um nun mal etwas anderes zu bringen, was die Probleme von einer neuen Seite zeigt, sondern weil das Theoretische selbst und als solches in ein Vor-theoretisches zurückweist"].

5 Se está pensando en Foucault, Lacan, Derrida, el pensamiento feminista de Judy Chicago, los estudios estéticos de los Gender Studies, Lesbian Studies, Feminist Studies o Queer Studies, entre tantos. En nuestra opinión, grosso modo, todos convergen en la consideración de que el ser del ente, como decía Nietzsche, es humo, una palabra vacía; luego, siguiendo la lógica más elemental, este ser hay que crearlo. Lo que aparece, sea cultural o no, responde a motivaciones de poder, artificios, voluntad soterrada de dominio. La bibliografía es bastante extensa. Baste mencionar: Butler, 1997, 135-159; 1993, 1-16 y 223-230; 1990; Jones, 1996 y Owens, 1985 , 93-124.

6 La mujer en el caso del feminismo, pero esto sería aplicable a cualquier fenómeno no ajustable con el patrón eidético usual y aceptado comúnmente, a los transexuales, a los parias sociales, a los vagabundos, etc. Fenómenos tales son harto interesantes, pues arrinconan al eidos en su propio límite, mostrándolo a veces para negarlo otras, a fin de lograr una ampliación, un ensanchamiento del propio eidos.

7 Crisis del eidos es un epítome que refleja la crisis de la filosofía, la crisis histórica, y la definición de los contornos del movimiento de la posmodernidad. Ver en: Lyotard, 2008. 
necesariamente, mentar un cambio originario en la forma específica de nuestro ser-en-elcuerpo. Una inversión del esencialismo metafísico, sigue siendo metafísica. La genealogía cultural, filosófica, practicada por estos movimientos sigue reivindicando una subjetividad hecha cogito, es decir, está en búsqueda de procesos "identitarios" al modo de las cogitationes cartesianas, impidiendo que salga a la luz esta relación intencional que implica el comportarse [sich-Verhalten]. No es que más bien, en la dualidad antropológica cartesiana, hayan una substancia pensante y otra corpórea, un alma y un cuerpo material, es que se permanece en la concepción de concebir al ser humano como ente al que le pertenece una subjetividad y, por tanto, es subiectum de algo, aunque lo sea de la creación de su propia identidad fabulada. El hecho es que se sigue pensando el mundo como un objeto [Gegenstand], como un algo-ahí que es puesto [setzend], fabulado o inventado por un sujeto, que, a pesar de ser débil y de no erigirse como cogito, coincide con éste en la imposibilidad de pensar la radicalidad de su ser-en-el-mundo. Lo que quiere mentar "en" y "mundo" quedan sin ser pensados, porque "en" no es una suerte de espacio físico, un contendor de espaciotiempo; ni "mundo" refleja lo externo de lo interno: el yo. Todo lo contrario, tanto "en" como "mundo" son dimensiones de nuestro propio modo de ser, nuestro ser comporta, de suyo, estas dimensiones existenciales. Por eso, para lograr una percepción cabal de nuestra subjetividad, es menester un esclarecimiento de estas dimensiones. La tarea no consiste en afirmar un nihilismo ontológico -ser es igual a humo-, sino en reclamar espacios de acogimiento para nuevas formas fenoménicas de corporalidad (ser-en-el-cuerpo) que se dan y se nos muestran. Ser-en-el-cuerpo, tener algo así como un cuerpo, un cuerpo que se comporta desde un "en" y un "mundo". ¿Qué es aquello que hace posible a un cuerpo? ¿qué es eso que hace posible la creación de nuevas identidades?

\subsection{El "en" como el suelo de lo abierto por el ser}

¿Cómo comenzamos en un cuerpo? En este sentido, sucedería algo similar a lo que Heidegger dice en el despliegue analítico de la estructura existencial del Dasein, cuando intenta describir el existenciario "ser-en" [In-sein], ya que no comenzamos siendo un "yo", un cogito, un género de pensamiento interiorizado en un cuerpo. No. Todo esto son abstracciones. Comenzamos siendo en lo dado culturalmente, en lo trasmitido por nuestras generaciones, en lo que se dice y se hace, en "lo uno" [das Man]:

Al principio no soy yo en el sentido del propio sí mismo, sino que soy los otros a la manera de lo impersonal. Desde éste y como éste me estoy, en primer lugar, dado a mí mismo $(1986,129)^{8}$.

Ante este aserto, pensado como descripción positiva de nuestro ser-en-el-mundo, debemos ser cautos y no considerarlo al modo psicológico. No es que yo comience siendo impersonal o inauténtico, para, a lo largo, hacerme a mí mismo. Infértil es toda la tarea que persigue esta tentativa, pues, entre otras cosas, no hay un esclarecimiento real, y fructífero,

8 "Zunächst bin nicht ich im Sinne des eigenen Selbst, sondern die Anderen in der Weise des Man. Aus diesem her und als dieses werde ich mir selbst zunächst gegeben". 
de lo que quiera decir sí-mismo". El "uno" heideggeriano muestra un fenómeno positivo; es una descripción de la forma en la que nos comportamos con las cosas, "en" el mundo; principio formal e indicativo de nuestra estructura ontológica. Con el "uno" se quiere mostrar el carácter originario de la espacialidad de nuestro modo de ser, que radica en un tener que atenerse a lo "ya" [schon] sido, acontecido. Según Heidegger, nosotros comenzando siendo en lo otro porque, justamente, lo otro ya se nos ha abierto. La tarea de la espacialidad será aquella que intente "abrir-espacio" [Raumgeben], un "ordenar espacio" [Einräumen] en nuestra constitución más propia: ser-en-el-mundo. Esta dimensión espacial es la que recoge la preposición "en", que hiende el horizonte de posibilidad para que las cosas se den y acontezcan. El "en" es la dimensión del "ahî" $[D a]$ del ser, porque es a partir de este "en" como las cosas se abren, devienen en fenómeno. Si el humano tiene cuerpo, lo tendrá en base a este "en", esto es, en relación a lo abierto en él. Por esta razón, el tener nunca es entendido como atributo de una interioridad hecha yo, ni mucho menos entendido como posesión de ésta, sino que tener significa la posibilidad de ser en lo abierto del ser.

Y una vez dicho esto, ¿qué tiene que ver lo dicho con la cuestión del cuerpo? El cuerpo, si lo entendemos materialmente -como Körper- , debemos hacerlo en relación a este "en", esto es, que no hay cuerpo vivido, ni material, que sea sin estar en relación a lo que aquí denominamos, fenomenológicamente, "en". Por eso, cuando decimos "somos" cuerpo, es necesario pensar la relevancia de ese "en", que configura, y explicita, el cómo, el dónde, el suelo, de todo lo que, en fenomenología, es llamado fenómeno, las cosas que se dan y aparecen. ¿Responde este "en" a una estructura cultural, de voluntad de poderío histórico?; ¿es el "en" constructo, una variante estructural del nexo público llamado habitus (Cfr. Bordieu, 1997, 87)? Iría contra el sano sentido común, negar que el complejo impersonal, aparato objetivo, del das Man, no respondiera a una determinación histórica concreta, en este caso, para occidente, de un predominio de la hegemonía masculina, pero de lo que se trata aquí es de ver cómo la corporalidad, y su acción, el "corporeizar" [leiben], no se co-determina en base a esta hegemonía histórica y cultural, sino al contrario: la corporalidad del Dasein está basada en la apertura del ser, una apertura que es vivida y proyectada como dimensión extática del "en". Este "en", por tanto, refleja la necesidad situacional de que, para el Dasein, tiene la apertura del ser, pues es el Dasein quien se relaciona con ella, soportándola, en un "proyecto" [Entwurf].

\subsection{La incomprensión del ser-en-el-cuerpo: el error de la construcción hegemónica}

Si ser-en-el-cuerpo mienta la relación originaria entre lo abierto por el ser y la forma en la que esta apertura es corporeizada o interiorizada, es decir, la forma en la que es encarnada, material y eidéticamente, no podemos afirmar que sea la determinación de la hegemonía histórica -sea machista o colonial- la que domina la forma de auto-conciencia ${ }^{10}$ que es nuestra corporalidad. Esta relación originaria de nuestra corporalidad, se ejerce en

9 Es que no se ha aclarado el sí mismo, no se ha hecho manifiesto. El sí mismo, según la visión de Heidegger, no se ha hecho fenómeno, y si no se ha hecho fenómeno, tendríamos que preguntarnos entonces: ¿es posible su conocimiento? Este será uno de los objetivos de Ser y Tiempo.

10 Decimos autoconciencia, no pensando en la capacidad epistemológica que para ella tiene un yo, sino para expresar que toda "corporalización" significa auto-conocimiento, conocimiento de sí mismo. Heidegger lo 
el suelo común de una situación ontológica denominada "en", y en la medida en que este "en" es el suelo en el que se tiene que desplegar, o proyectar, nuestro ser-en-el-cuerpo, es menester salvar esta situación. En este caso, con Ortega y Gasset, podríamos parafrasear lo dicho: "si no salvo a las circunstancias..." (Cfr. 1966, 322). Imperativo, para todo aquel que existe, es salvar este "en", para poder salvarse a sí mismo. En innumerables ocasiones, se le ha acusado a Heidegger de apelar a una ética de la decisión (Cfr. Krämer, 1992; Luckner, 2001, 167; Volpi, 2009, 3-33; 1994, 195-212 y 1988, 1-44), en relación a la tarea dual planteada en Ser y Tiempo: el Dasein es aquel ente que tiene la posibilidad de vivir una vida en propiedad [Eigentlichkeit], o dejarse caer en la impropiedad [Uneigentlichkeit]. ¿Se podría decir lo mismo con la corporalidad, esto es, hay una manera de vivir propiamente mi cuerpo con respecto a otra impropia? En este caso, podríamos decir que sí. Justamente, aquí se asienta una de las críticas heideggerianas al cogito cartesiano que, en virtud de su dualismo metafísico entre res extensa y res cogitans, hace que el ser humano huya, evada y banalice la cuestión de la relación con su propia apertura y el modo en la que éste se relaciona con ella, es decir, como un comportarse. La lectura de Heidegger hace ver, por ejemplo en el caso de Descartes ( $C f r$. 1986, 41-45 y 83-95), que la experiencia del cuerpo como mezcolanza entre substancia pensante y substancia corpórea, es una forma de soslayar el principio originario de la corporalidad, en su relación con lo abierto por el ser (lo no-ente ${ }^{11}$ ) en su situación ontológica: el "en” y el mundo. Por esta razón, aunque se parta de la descripción positiva heideggeriana sobre el modus vivendi cotidiano del Dasein, en la estructura impersonal del das Man, esto no quiere decir que éste sea el principio por el que se rija nuestro ser-en-el-cuerpo. Lo que se persigue con esta constatación es hacer notar que empezamos "entregados" [überantworten] en lo otro, en un estado a-teórico, a leguas de las consideraciones primigenias del pensamiento occidental: alma, persona, cogito, yopensante, etc. Lo propio de nuestro ser es estar, estar-entregado a nuestro modo ser, un modo de ser que se define como posibilidad, dentro de un horizonte fáctico, finito y, además, en el cumplimiento de su propio telos: "ser-para-la-muerte" [sein-zum-Tode]. Por todo ello, no nos resulta fructífera la visión de cierta parte del pensamiento filosófico de la segunda mitad del siglo XX, y en concreto del feminismo, pues, desde su lectura innegable de Heidegger, pensamos que no han comprendido los entresijos del meditar de éste, a no hacer constar la relevancia de la apertura y la dimensión situacional del "en", el mundo y el "ahî" $[D a]$. Una relevancia no surcada, primordialmente, por los elementos históricos que toda cultura comporta, ora en su relevancia de dominación, ora en la dimensión puramente epocal. La pregunta que tenemos que resolver es si, desde esta situación, podemos aventurar una forma de vivir en propiedad nuestro ser-en-el-cuerpo.

\section{La tarea de la apropiación del ser-en-el-cuerpo}

En el mundo líquido que vivimos (Cfr. Bauman, 2013), las relaciones personales están cada vez más orientadas a la lógica del capitalismo. Hasta la propia percepción intelectual

piensa con el término "estar-despierto" [Waschsein], para significar el estado de alerta que el Dasein debe tener ante su tendencia natural a la "caída" [Fallen].

11 Entiéndase: lo no-ente, en virtud de la diferencia ontológica, el ser no es un ente. 
y universitaria es tomada en tanto capital humano ${ }^{12}$, es decir, como un elemento susceptible de conversión económica. Para decirlo en palabras de Heidegger, esta situación es nuestro propio das Man. En base a la relación con nuestro cuerpo y más allá de tendencias seculares -como el culto del cuerpo físico en los gimnasios, la cultura Fitness- la inmensa mayoría de nosotros, europeos, nos seguimos comportando en base a la dualidad cartesiana: alma y cuerpo, es decir, hemos sido incapaces de, según Heidegger, explorar nuestra propia "mismidad" [Selbstheit]. Así, de este modo, el adolescente acude presto al gimnasio para tonificar su cuerpo, a fin de que, más pronto que tarde, pueda embriagar a una joven, extraordinariamente guapa -o a la inversa, también- ¿Qué es, entonces, esto de vivir propiamente nuestro ser-en-el-cuerpo? Con vivir propiamente nuestro ser-en-el-cuerpo se quiere expresar un contra-movimiento naciente del movimiento inercial de nuestro "estado de caída" [Verfallen] en el "uno" [Man].

A partir de aquí, entonces, nos limitaremos a esbozar un serie de tentativas, o campos de futura explotación meditativa, para realizar una comprensión más particular de lo que proponemos como modo propio de ser-en-el-cuerpo.

\subsection{Propedéutica: conoce tu ser-en-el-cuerpo}

El ser-en cobra el modo existencial del no-ser-en-casa. Es lo que quiere decir al hablar de lo "inhóspito" [...] El no-ser-en-casa acaba de ser concebido ontológicoexistencialmente como el fenómeno más originario (Heidegger, 1986, 212 ${ }^{13}$ ).

El Dasein es un ser-en-el-mundo, es decir, existe arrojado en su propio ser-posible y además lo hace entregado a un mundo. Quiere esto decir, también, que al Dasein le pertenece ontológicamente un "ser-en" [In-sein]. Dentro de la hermenéutica fenomenológica de Heidegger, el Dasein está "familiarizado con" [Vertarautsein mit] su "mundo entorno" [Umwelt], este mundo le otorga al Dasein la brújula con la que dirigir su vida; el mundo cotidiano es, para el Dasein, un "ser-en-casa ${ }^{14 "}$ [Zuhause-sein]. De suerte que con la corporalidad sucede algo similar, las propuestas auto-perceptivas que una determinada cultura tiene para sí, dotan al ser humano de una imagen propia, en sus diversos horizontes hermenéuticos: el cuerpo es la cárcel del alma, el cuerpo como pecado y castigo, el cuerpo virtual, el cuerpo soñado, proyectado idealmente y programado por la moda, el marketing estético, etc. Con Heidegger, podríamos decir, que todas estas formas son modos impersonales de ser-en-elcuerpo, pues son concepciones heredaras que no "transparentan" [Durchsichtigkeit] nuestro propio modo de ser. La tarea de la apropiación de nuestra corporalidad consiste, entonces,

12 La tendencia a considerar la inteligencia humana como capital humano es un tema que requiere tiempo y demora. Por razones evidentes, el lector entenderá que no pueda extenderme. Sin embargo, piénsese las relaciones entre el capital humano y la ciencia entendida modernamente como poder. Véase, por ejemplo, el siguiente informe del CES: (Consejo Económico y Social, 2009).

13 "Das In-sein kommt in den existenzialen Modus des Un-zuhause. Nichts anderes meint die Rede von der Unheimlichkeit [...] Das Un-zuhause muß existencial-ontologisch als das ursprünglichere Phänomen begriffen werden".

14 Recordemos lo que dijo el poeta Novalis acerca de la tarea del filosofar: "La filosofía es propiamente nostalgia, un impulso de estar dondequiera en casa". ["Die Philosophie ist eigentlich Heimweh, ein Trieb überall zu Hause zu sein"] $(1987,491)$. 
en convertir nuestra relación situacional de nuestro cuerpo "en" el "mundo", en una forma que responda a eso que somos, en primer lugar: entes que están entregados a las cosas que se le presentan, circundados por la finitud, la facticidad, destinados a morir. ¿Y cómo se logra esto? Atreviéndose a reclamar para sí, en los términos ontológicos-existenciales que lo hace Heidegger, lo "inhóspito" [Umheimlichkeit], esto es, que la evidencia apodíctica de nuestro modo de ser consiste en relacionarse con lo abierto de las cosas, con aquellas cosas que, en virtud de la diferencia ontológica, son inteligibles y unificadas por el "ser". Este ser, además, tiene la connotación de lo inhóspito porque, según su definición, no se ajusta a un ente, es un principum no asignable a un principatum. En términos hermenéuticos, es un horizonte de significatividad no conformado en alguna determinación específica y concreta, sino que sirve de fundamento para toda determinación posible. En el caso del ser-en-el-cuerpo, la tentativa estribaría en la posibilidad de una corporalidad que acoja en su seno a esta apertura, en sus consecuencias más radicales, en su inconformidad bajo alguna determinación particular, óntica. ¿Es ello realmente posible?

Lo realmente decisivo para el interés filosófico del mismo es que el mero hecho de pensar en la posibilidad de que nuestro modo de ser es un comportarnos con las cosas, hace que cambiemos nuestra autopercepción y que comprendamos porqué la visión occidental de nuestra corporalidad, ejemplarizada en la dualidad alma y cuerpo, es una modificación existencial de la relación originaria mentada como ser-en-el-mundo. De este modo, comprenderemos el equívoco, por ejemplo, de la percepción feminista, pues la lucha no debe estar en la dislocación de ideologías "reificantes" que petrifican las relaciones sexuales en una estructura de dominación (Cfr. Butler, 1989, 86), antes al contrario, construir una propedéutica cuya finalidad sea el esclarecimiento de nuestra estructura existencial y ontológica. En este sentido, la filosofía tiene mucho que decir. La creación de esta propedéutica ha de ser un compromiso interdisciplinar. Porque la tarea no consiste en inventar otras hegemonías más amables para que, por así decir, sigamos siendo cartesianos en hegemonías negadores de toda substancialidad, es decir, siendo "malos" cartesianos.

Nuestro ser-el-el-cuerpo comienza en nuestro ser-en-el-mundo y si no salvamos nuestro "en", no podremos salvar nuestro cuerpo.

\section{Conclusión: en búsqueda del ser-en-el-cuerpo}

La presente meditación no ha buscado, en ningún momento, la consecución de respuestas y conclusiones absolutas. Precisamente, la naturaleza de la cuestión pensada -cómo aparece el cuerpo entendido como ser-en-el-cuerpo- lo impide. Aun así, pasaremos a resumir brevemente, de forma indicativa, lo alcanzado.

Ante la pregunta cómo se da un cuerpo, éste no se da, inmediatamente, como alma, substancia o cogito, sino como "un comportarse" [ein-sich-Verhalten]. El comportarse, que define nuestro modo de ser -también en tanto temple anímico- es siempre un comportarse "en" y un "mundo", que es "ahî" $[D a]$ en lo abierto del ser [Erschlossenheit]. Por tanto, hay que dilucidar, primero, si se quiere saber qué es el cuerpo, estas dimensiones ontológicas originarias.

Por otro lado, el "en" es el cómo, el dónde, el suelo, de todo lo que, en fenomenología, es llamado fenómeno, las cosas que se dan y aparecen. El mundo es lo abierto en este "en". Toda corporalidad, si realmente "es", ha de ser dentro del horizonte del "en" y el mundo. 
Estos horizontes no responden, de modo originario, a constructos hegemónicos, estructuras de voluntad de poder de cierto correlato histórico. Más bien, éstos son modificaciones de la experiencia originaria que, en Heidegger, tiene un nombre: lo abierto por el ser.

También, el feminismo, en particular, se equivoca con el propósito de considerar el cuerpo, el sexo y el género, como construcciones hegemónicas, pues, si así lo hacen, siguen viviendo en el paradigma de la modernidad, es decir, cartesiano, como modificación ciega ante el fundamento de la corporalidad: lo abierto por el ser.

Por último, la tarea sería, más bien, la de una propedéutica filosófica, en un diálogo interdisciplinar con otras ciencias, cuya finalidad sea transparentar [Durchsichtigkeit] nuestro propio modo de ser.

\section{Bibliografía}

Bauman, Zygmunt (2013): La cultura en el mundo de la modernidad líquida, Fondo de Cultura Económica, México.

Bravo, Cristián (2014): Ética a Nicómaco. Libro II (1103 a 14 - 1109 b 29), in Id., Apeiron: Estudios de Filosofía, Madrid, n 1, pp. 382-412.

Bordieu, Pierre (1997): Outline of a Theory of Practice, Cambridge University Press, Nueva York.

Butler, Judith (1997): Excitable Speech. Apolitics of the Performative, Routledge, LondresNueva York.

Butler, Judith (1993): Bodies That Matter. On the Discursive Limits of Sex, Routledge, Londres-Nueva York.

Butler, Judith (1990): Gender Trouble. Feminism and the Subversion of Identity, Routledge, Londres-Nueva York.

Butler, Judith (1989): Sexual Ideology and Phenomenological Description: A Feminist Critique of MerleauPonty's Phenomenology of Present, in Id., The Thinking Muse: Feminism and Modern French Philosophy, Indiana University Press, Bloomington, pp. 85-100.

Consejo Económico y Social (2009): Sistema educativo y capital humano, Consejo Económico y Social, Madrid. http://www.ces.es/documents/10180/18510/Inf0109.

Descartes, René (1988): Discurso del método, Losada, México.

Heidegger, Martin (2006): Zollikoner Seminare, II. Abteilung, Band 89, Vittorio Klostermann, Frankfurt am Main.

Heidegger, Martin (1999): Die Idee der Philosophie und das Weltanschauungsproblem (Kriegsnotsemester 1919, in Id., Zur Bestimmung der Philosophie, II. Abteilung, Band 56/57, Vittorio Klostermann, Frankfurt am Main, pp. 3-117.

Heidegger, Martin (1989): Nietzsche I, Neske, Pfullingen.

Heidegger, Martin (1986): Sein und Zeit, Max Niemeyer, Tübingen. [(1997): Ser y Tiempo, trad., Jorge Eduardo Rivera, Editorial Universitaria S.A., Santiago de Chile].

Heidegger, Martin (1976): Metaphysische Anfangsgründe der Logik im Ausgang von Leibniz, II. Abteilung, Band 26, Vittorio Klostermann, Frankfurt am Main

Husserl, Edmund (1952): Ideen zu einer reinen Phänomenologie und phänomenologischen Philosophie (Volumen Segundo: Phänomenologische Untersuchungen zur Konstitution) (Husserliana IV), Martinus Nijhoff, Den Haag. 
Irigaray, Luce (1984): Ethique de la difference sexuelle, Minuit, Paris.

Irigaray, Luce (1983), L'Oubli de l'aire chez Heidegger, París, Minuit.

Jones, Amelia (1996): The Sexual Politics of the Dinner Party. A Critical Context, in Id., Sexual Politics. Judy Chicago's Dinner Party in Feminism Art History, University of Chicago Press, Berkeley, pp. 88-102.

Krämer, Hans (1992): Integrative ethic, Suhrkamp, Frankfurt am Maim.

Novalis, Friedrich (1987): Aus dem Allgemeinen Brouillon, in Id., Novalis: Werke, Beck, München.

Luckner, Andreas (2001): Wie es ist, selbst zu sein. Zum Begriff Eigentlichkeit, in Id., Sein und Zeit, Akademie Verlag, Berlin, pp. 149-168.

Lyotard, Jean-François (2008): La posmodernidad explicada a niños, Gedisa Barcelona.

Ortgea y Gasset, José (1966): Meditaciones del Quijote, in Id., Obras Completas, vol. I., Revista de Occidente, Madrid.

Owens, Craig (1985): El discurso de los otros: las feministas y el posmodernismo, in Id., La posmdernidad, Paidós, Barcelona, pp. 93-124.

Rubin, Gayle (1984): The Traffic in Women. Notes on the Political Economy of Sex, in Id., Pleasure and Danger: Exploring Female Sexuality, Routledge, Boston, pp. 157-210.

Volpi, Franco (2009): Ser y tiempo: ¿una versión modernizada de la Ética a Nicómaco?, in Id., Martin Heidegger. La experiencia del camino, Uninorte, Barranquilla, pp. 3-31.

Volpi, Franco (1994): Being and time: A translation of the Nichomachean Ethics? , in Id., Reading Heidegger from the start. Essays in his earliest thought, State University of New York Press, Albany, pp. 195-212.

Volpi, Franco (1988): Dasein como praxis. L'assimilation et la radicalisation heideggeriene de la philosophie practique d'Aristotle, in Id., Heidegger et l'idée de la phenomenology, , Kluwer Academic Publisher, Dordrecht, pp. 1-44. 
